\title{
A Weighted Least-Squares Approach to Parameter Estimation Problems Based on Binary Measurements
}

\author{
Eric Colinet, Jérôme Juillard, Member, IEEE
}

\begin{abstract}
We present a new approach to parameter estimation problems based on binary measurements, motivated by the need to add integrated low-cost self-test features to microfabricated devices. This approach is based on the use of original weighted least-squares criteria: as opposed to other existing methods, it requires no dithering signal and it does not rely on an approximation of the quantizer. In this paper, we focus on a simple choice for the weights and establish some asymptotical properties of the corresponding criterion. To achieve this, the assumption that the quantizer's input is Gaussian and centered is made. In this context, we prove that the proposed criterion is locally convex and that it is possible to use a simple gradient descent to find a consistent estimate of the unknown system parameters, regardless of the presence of measurement noise at the quantizer's input.
\end{abstract}

Index Terms-parameter estimation, quantized observations, binary sensors, FIR digital filters.

\section{INTRODUCTION}

$\mathrm{I}^{\mathrm{N}}$ $\mathrm{N}$ this paper, we present a new parameter estimation method based on binary measurements. This work was originally motivated by the need to add integrated low-cost self-test features to microfabricated devices, such as MEMS and NEMS. Even though there exists a wide range of applications where identification methods based on binary observations are necessary or desirable [1], the focus is brought here on the test of microelectronic devices. It is wellknown that, as characteristic dimensions become smaller, the dispersions afflicting electronic devices tend to become larger. Typical sources of uncertainty and dispersion are variations in the fabrication process, changes in the operating conditions or imperfect knowledge of physics. As a consequence, it is usually impossible to guarantee a priori that a given device will function properly. Expensive tests must then be run after fabrication to ensure that only suitable devices are commercialized. Furthermore, self-test (and self-tuning) features, such as parameter estimation routines, must often be implemented, so that devices can adapt to changing conditions.

E. Colinet is with CEA-LETI, MINATEC, 17 rue des Martyrs, 38054 Grenoble Cedex 9, France (+33-4-38-78-11-40, eric.colinet@cea.fr).

J. Juillard is with SUPELEC, SSE, 3 rue Joliot-Curie, 91192 Gif-surYvette Cedex, France (+33-1-69-85-14-08, jerome.juillard@supelec.fr).
However, most parameter estimation methods [2-3] rely on high-resolution digital measurements. Their integration requires the implementation of high-resolution analog-todigital converters (ADCs) and, thus, results in longer design times, larger silicon areas and increased costs. Our objective is then to develop a parameter estimation method that relies on very low-resolution (ideally binary) measurements, in order to keep the added cost of testing as small as possible.

In the field of micro-electronics, the issue of parameter estimation of linear systems from binary data has partially been addressed by Negreiros [4] and Juillard and Colinet [5] $]^{1}$. In [5], a white Bernoulli input is used to excite the unknown system. Provided the impulse response does not vanish too quickly, it is possible to establish an analytical relationship between the cross-covariance function of the binary outputs and inputs and the impulse response of the unknown system and, thus, to identify the system.

Outside the context of micro-electronics, the most significant contributions come from Wigren [6], Wang [1,7-8], Rafajlowicz [9-10] and their co-workers. In [9-10], the author considers a linear system excited by a deterministic signal. The output of the system is measured via a quantizer whose threshold is "randomly specified"2. The author then uses an elegant argument based on Von Neumann's theorem to estimate the Fourier transform of the quantizer's input from the quantized data and, knowing the inputs, it becomes simple to estimate the transfer function of the system. In [1], Wang et al. introduced a new method for estimating parameters from binary (or quantized) data. The unknown system is excited by a periodic signal and, as in Rafajlowicz's work, the threshold of the quantizer is randomly specified. However, the hypotheses on the quantizer's input and on the random threshold are much less strict: the major constraint is that the cumulative distribution function (cdf) of the threshold must be invertible. The parameter estimation problem then reduces to solving a linear system. This approach is generalized in [7],

\footnotetext{
${ }^{1}$ The approach in [5] was actually developed as a "quick and dirty" way to initialize the optimization algorithms on which the method presented in section III relies. However, because of its minimal requirements, it can also be used as a standalone parameter estimation approach.

${ }^{2}$ From the point of view of electronics, this randomly specified threshold can be implemented as an addition of a dithering signal at the input of the (fixed-threshold) quantizer.
} 


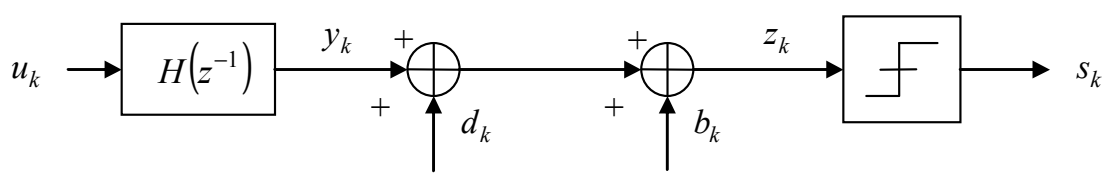

Fig. 1 - Framework and notations.

where it is shown that the cdf of the threshold can be estimated along with the parameters of the system, and in [8] where the identification of infinite impulse response (IIR) systems and nonlinear Wiener systems is addressed. Finally, Wigren has developed a least mean squares (LMS) approach to the problem of online parameter estimation from binary observations [6]. This method is based on the definition of an approximate gradient of the (quantized) least-squares criterion. Under some stationarity hypotheses and in the absence of measurement noise at the quantizer's input, it is possible to guarantee the asymptotical convergence of this method to the nominal parameters.

Each of these methods has its merits and weaknesses. It is our opinion that Wigren's approach is the one that fits better in the context of test of electronics system: as opposed to Wang's or Rafajlowicz's methods, it does not require a varying threshold (dithering signal), which can only be implemented with a high-resolution DAC or a surface-consuming analog component. Furthermore, it can be used online as well as offline. However, it does rely on an approximation of the quantizer (and, thus, it is not an exact method, stricto sensu, as opposed to [1]). Moreover, convergence is not guaranteed in the presence of measurement noise at the quantizer's input.

The weighted least-squares (WLS) method we introduce in the present paper bears some likeness to Wigren's, in the sense that it is also based on the minimization of a least-squares criterion. Its distinguishing features are that no approximation of the quantizer is made and that convergence can be guaranteed, even in the presence of measurement noise. Furthermore, this approach is more general than [1] in the sense that it does not require the presence of noise at the quantizer input.

The outline of the article is the following. In section II, the framework and the notations are introduced. In section III, we show how to construct exact WLS continuous criteria for parameter estimation from binary data. The asymptotic properties (when the number of data goes to infinity) of one of these criteria are established in section IV. Using probabilistic arguments, it is shown that this approach leads to a consistent estimate of the nominal parameters of the system, even in the presence of measurement noise. Section V contains some concluding remarks.

\section{FRAMEWORK AND NOTATIONS}

Let us consider a discrete-time linear time-invariant (LTI) system $H$. We assume $H$ has a finite impulse response of length $L$, i.e. the impulse response can be represented by a column vector $\boldsymbol{\theta}=\left(\theta_{k}\right)_{k=1}^{L}$. Let $u_{l}$ be the known scalar value of the system input at time $l$ and $y_{l}$ be the (scalar) value of the system output, so that:

$y_{l}=\boldsymbol{\varphi}_{l, L}^{T} \boldsymbol{\theta}$,

where $\boldsymbol{\varphi}_{l, L}=\left(u_{k}\right)_{k=l-L+1}^{l}$ is the $L$ sample-long (column) regression vector at time $l$. Let $d_{l}$ be an additive dithering signal at the system output and $b_{l}$ an additive noise (in other words, $d_{l}$ is known whereas $b_{l}$ is not). Let $m_{x}$ and $\sigma_{x}{ }^{2}$ denote the first- and second-order moments of any signal $x$. The system output is measured via a 1-bit ADC so that only the sign $s_{l}=S\left(z_{l}\right)$ of the system output is known, where

$$
\begin{aligned}
& \left\{\begin{array}{l}
S(x)=1, \text { if } x \geq 0 \\
S(x)=-1, \text { otherwise }
\end{array},\right. \\
& \text { and } z_{l}=y_{l}+d_{l}+b_{l} .
\end{aligned}
$$

We are interested in finding an offline estimate $\hat{\boldsymbol{\theta}}$ of $\boldsymbol{\theta}$, based on $N$ observations of $u_{l}, s_{l}$ and $d_{l}$. Let us also introduce $\hat{y}_{l}$, the estimated system output, $\hat{z}_{l}=\hat{y}_{l}+d_{l}=\boldsymbol{\varphi}_{l, \hat{L}}^{T} \hat{\boldsymbol{\theta}}_{l}+d_{l}$, the estimated input of the comparator, and $\hat{s}_{l}=S\left(\hat{z}_{l}\right)$. These notations are summed up in Fig. 1. Finally, the notation $\left.\boldsymbol{\theta}\right|_{\hat{L}}$ designates:

- $\boldsymbol{\theta}$ padded with $\hat{L}-L$ zeros if $\hat{L}>L$,

- $\quad \boldsymbol{\theta}$ truncated to length $\hat{L}$, if $\hat{L}<L$.

\section{DEFINITION OF WLS CONTINUOUS CRITERIA}

In the following sections, we concentrate on criteria of the form:

$J_{p}^{N}=\frac{1}{4} \frac{\sum_{l=1}^{N} \hat{z}_{l}^{2 p}\left(s_{l}-\hat{s}_{l}\right)^{2}}{\sum_{l=1}^{N} \hat{z}_{l}^{2 p}}, p \geq 1$.

Note that taking $p=0$ in (3) yields $J_{0}^{N}=\frac{1}{4 N} \sum_{l=1}^{N}\left(s_{l}-\hat{s}_{l}\right)^{2}$, the "classical" unweighted least-squares criterion. However, because of the quantization of $s_{l}$ and $\hat{s}_{l}, J_{0}^{N}$ is piecewiseconstant with respect to $\hat{\boldsymbol{\theta}}$ (and, thus, not easily amenable to optimization). In the numerator of (3), the term $\hat{z}_{l}{ }^{2 p}$ acts as a (positive) weight to the binary-valued error $\left(s_{l}-\hat{s}_{l}\right)^{2}$. Since $\hat{z}_{l}{ }^{2 p}$ is continuous and is equal to 0 when $\hat{s}_{l}=S\left(\hat{z}_{l}\right)$ changes, $\hat{z}_{l}^{2 p}\left(s_{l}-\hat{s}_{l}\right)^{2}$ is a continuous function of $\hat{\boldsymbol{\theta}}$ and so is $J_{p}^{N}$. Moreover, $\hat{z}_{l}{ }^{2 p}\left(s_{l}-\hat{s}_{l}\right)^{2}$ is continuously differentiable with 
respect to $\hat{z}_{l}$. Since $\hat{z}_{l}(\hat{\boldsymbol{\theta}})$ is continuously differentiable everywhere, $J_{p}^{N}(\hat{\boldsymbol{\theta}})$ is also continuously differentiable everywhere, except when:

$\sum_{l=1}^{N} \hat{z}_{l}^{2 p}=0 \Leftrightarrow \hat{z}_{l}=0,1 \leq l \leq N \Leftrightarrow\left[\begin{array}{c}\boldsymbol{\varphi}_{1, \hat{L}}^{T} \\ \vdots \\ \boldsymbol{\varphi}_{N, \hat{L}}^{T}\end{array}\right] \hat{\boldsymbol{\theta}}+\left[\begin{array}{c}d_{1} \\ \vdots \\ d_{N}\end{array}\right]=\mathbf{0}$.

Provided $N>\hat{L}$ and under some mild assumptions on $u_{l}$, there usually exists no $\hat{\boldsymbol{\theta}}$ that verifies $(4)^{3}$. The major exception is when $d_{l}=0,1 \leq l \leq N$, in which case (4) has $\hat{\boldsymbol{\theta}}=\mathbf{0}$ for solution, regardless of $u_{l}{ }^{4}$. Note that $0 \leq J_{p}^{N} \leq 1$.

Thus, it is possible to construct continuously differentiable WLS criteria for parameter estimation problems based on binary observations without having to rely on an approximation of the quantizer. These WLS criteria are "exact" in the sense that $J_{0}^{N}=0 \Rightarrow J_{p}^{N}=0^{5}$ and they can be minimized using any standard optimization method [2]. Note that it is impossible to guarantee their convexity a priori, without further assumptions on the inputs.

In [11], Bai shows that minimizing $J_{0}^{N}$ yields a consistent estimation of $\boldsymbol{\theta}$, supposing $u$ is white, $b=d=0, L=\hat{L}$ and $N$ goes to infinity. Since $J_{0}^{N}=0 \Rightarrow J_{p}^{N}=0$, it follows that the same is true of $J_{p}^{N}$. In the next section, we establish asymptotic properties of $J_{1}^{N}$ under different assumptions, some less restrictive than in [11] $(L \neq \hat{L}, b \neq 0, d \neq 0$, coloured input), some not (Gaussianity of $\hat{z}$ and $z$ ).

\section{ASYMPTOTIC PROPERTIES OF $J_{1}^{N}(\hat{\boldsymbol{\theta}})$}

\section{A. Asymptotic expression of $J_{1}^{N}(\hat{\boldsymbol{\theta}})$}

In order to perform the asymptotic analysis of $J_{1}^{N}$, some assumptions must be made. A1- $u_{l}, d_{l}$ and $b_{l}$ are stationary ergodic Gaussian processes (not necessarily white) with mean 0. $J_{1}^{N}$ can then be interpreted as the ratio of empirical means of stationary ergodic processes:

$J_{1}^{N}=\frac{1}{4 N} \sum_{l=1}^{N} \hat{z}_{l}^{2}\left(s_{l}-\hat{s}_{l}\right)^{2} / \frac{1}{N} \sum_{l=1}^{N} \hat{z}_{l}^{2}$,

\footnotetext{
${ }^{3}$ In a deterministic context, it suffices to design the input so that the first $\hat{L}+1$ lines of (4) are independent. In a stochastic context, one can find simple conditions under which (4) has no solution with probability 1.

${ }^{4}$ Note that these properties also hold when one replaces $\hat{z}_{l}^{2 p}$ by $f\left(\hat{z}_{l}\right)$ in (3), where $f$ is any positive even continuously-differentiable function such that $f(0)=0$.

${ }^{5}$ If $\hat{z}_{l}$ is a continuous random variable, one can also prove that $J_{p}^{N}=0 \Rightarrow J_{0}^{N}=0$ with probability 1 .
}

Thus:

$J_{1}^{\infty}=\lim _{N \rightarrow \infty} J_{1}^{N}=\frac{1}{4} \frac{\mathrm{E}\left(\hat{z}^{2}(s-\hat{s})^{2}\right)}{\mathrm{E}\left(\hat{z}^{2}\right)}$

From A1, $\hat{z}$ and $z$ are jointly Gaussian with mean $0^{6}$. One may then write:

$J_{1}^{\infty}=\frac{1}{4 \sigma_{\hat{z}}^{2}} \int_{-\infty-\infty}^{\infty} \int_{-\infty}^{\infty} \hat{z}^{2}(S(\hat{z})-S(z))^{2} p(\hat{z}, z) d \hat{z} d z$

$=\frac{1}{4 \sigma_{\hat{z}}^{2}}\left(\int_{-\infty}^{0} \int_{-\infty}^{\infty} \hat{z}^{2} p(\hat{z}, z) d \hat{z} d z+\int_{0}^{\infty} \int_{-\infty}^{0} \hat{z}^{2} p(\hat{z}, z) d \hat{z} d z\right)^{\prime}$

$p(\hat{z}, z)=\frac{\exp \left(\frac{-1}{2\left(1-r^{2}\right)}\left(\frac{\hat{z}^{2}}{\sigma_{\hat{z}}{ }^{2}}+\frac{z^{2}}{\sigma_{z}^{2}}-\frac{2 r \hat{z} z}{\sigma_{\hat{z}} \sigma_{z}}\right)\right)}{2 \pi \sigma_{\hat{z}} \sigma_{z} \sqrt{1-r^{2}}}$,

where $r$ is the cross-correlation coefficient of $z$ and $\hat{z}$. (7) can be expressed as:

$J_{1}^{\infty}=\frac{1}{\pi}\left(\operatorname{acos}(r)-r \sqrt{1-r^{2}}\right)$.

The same reasoning applies to the classical least-squares criterion. This yields:

$J_{0}^{\infty}=\lim _{N \rightarrow \infty} J_{0}^{N}=\frac{1}{\pi} \operatorname{acos}(r)$.

$J_{1}^{\infty}$ is a decreasing convex function of $r$, for $r \in[0,1]$, whereas $J_{0}^{\infty}$ is decreasing and concave on the same interval (Fig. 2).

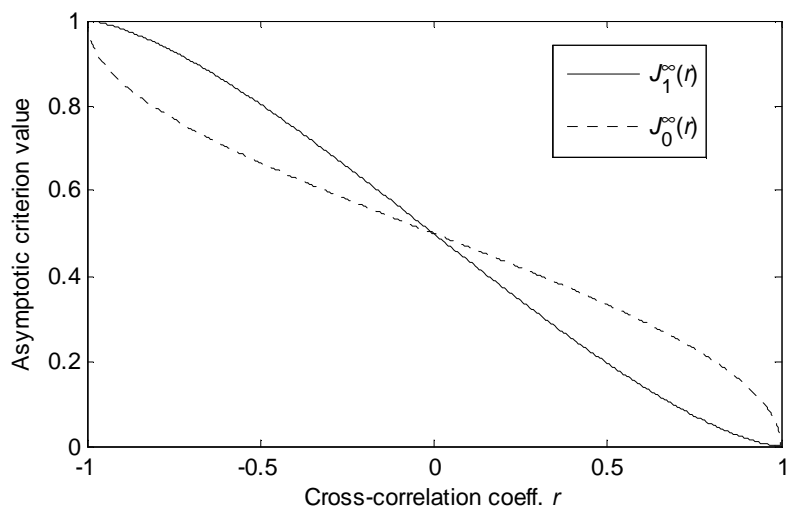

Fig. 2 - Comparison of $J_{0}^{\infty}(r)$ and $J_{1}^{\infty}(r)$.

B. Properties of $J_{1}^{\infty}(\hat{\boldsymbol{\theta}})$

The following assumptions must be added to A1: A2- $d_{l}$ and $b_{l}$ are white. A3- $u_{l}, d_{l}$ and $b_{l}$ are independent. Defining $\mathbf{R}_{u}^{P Q}$ the $P \times Q$ covariance matrix of $\boldsymbol{\varphi}_{., P}$ and $\boldsymbol{\varphi}_{., Q}$, we also assume : A4- $\mathbf{R}_{u}^{\hat{L} \hat{L}}$ has full rank. The following theorem can then be established.

Theorem - If A1, A2, A3 and A4 hold, the following properties are true:

\footnotetext{
${ }^{6}$ In practice, the joint Gaussianity of $\hat{z}$ and $z$ may be approximately achieved with less stringent assumptions on the distribution of the system input
} 
Property 1 - If $\hat{L} \geq L$,

a) if ${\sigma_{d}}^{2} \neq 0$, the minimum of $J_{1}^{\infty}$ is unique and it is reached for $\hat{\boldsymbol{\theta}}=\left.\boldsymbol{\theta}\right|_{\hat{L}}$.

b) if ${\sigma_{d}}^{2}=0, J_{1}^{\infty}$ has infinitely many minima of the form $\left.\hat{\boldsymbol{\theta}}=\left.\kappa \boldsymbol{\theta}\right|_{\hat{L}}, \kappa \in\right] 0,+\infty[$.

Property 2 - If $\hat{L}<L$ and $u$ is white, Property 1 still holds.

Property $3-J_{1}^{\infty}(\hat{\boldsymbol{\theta}})$ is convex in a neighbourhood of its minimum (minima).

Property 4 - Properties 1 and 2 hold for $J_{0}^{\infty}(\hat{\boldsymbol{\theta}})$.

Proof - The cross-correlation coefficient of two centred variables is $r(\hat{\boldsymbol{\theta}})=\mathrm{E}(z \hat{z}) /\left(\sigma_{z} \sigma_{\hat{z}}\right)$. Under A2-A3, $r(\hat{\boldsymbol{\theta}})$ can be expanded into:

$$
r(\hat{\boldsymbol{\theta}})=\frac{1}{\sqrt{\sigma_{b}{ }^{2}+\sigma_{d}{ }^{2}+\boldsymbol{\theta}^{T} \mathbf{R}_{u}^{L L} \boldsymbol{\theta}}} \frac{\sigma_{d}{ }^{2}+\boldsymbol{\theta}^{T} \mathbf{R}_{u}^{L \hat{L}} \hat{\boldsymbol{\theta}}}{\sqrt{\sigma_{d}{ }^{2}+\hat{\boldsymbol{\theta}}^{T} \mathbf{R}_{u}^{\hat{L} \hat{L} \hat{\boldsymbol{\theta}}}}} .
$$

The extrema of $r(\hat{\boldsymbol{\theta}})$ are given by differentiating (11):

$$
\frac{\partial r}{\partial \hat{\boldsymbol{\theta}}}=\mathbf{0} \Leftrightarrow\left(\sigma_{d}{ }^{2}+\hat{\boldsymbol{\theta}}^{T} \mathbf{R}_{u}^{\hat{L} \hat{L}} \hat{\boldsymbol{\theta}}\right) \mathbf{R}_{u}^{\hat{L} L} \boldsymbol{\theta}=\left(\sigma_{d}{ }^{2}+\boldsymbol{\theta}^{T} \mathbf{R}_{u}^{L \hat{L}} \hat{\boldsymbol{\theta}}\right) \mathbf{R}_{u}^{\hat{L} \hat{\boldsymbol{\theta}}} \hat{\boldsymbol{\theta}} .
$$

Thus, provided A4 holds and $\sigma_{d}{ }^{2}+\boldsymbol{\theta}^{T} \mathbf{R}_{u}^{L \hat{L}} \hat{\boldsymbol{\theta}} \neq 0$,

$\hat{\boldsymbol{\theta}}=\frac{\sigma_{d}{ }^{2}+\hat{\boldsymbol{\theta}}^{T} \mathbf{R}_{u}^{\hat{L} \hat{L}} \hat{\boldsymbol{\theta}}}{\sigma_{d}{ }^{2}+\boldsymbol{\theta}^{T} \mathbf{R}_{u}^{L \hat{L}} \hat{\boldsymbol{\theta}}} \mathbf{R}_{u}^{\hat{L} \hat{L}^{-1}} \mathbf{R}_{u}^{\hat{L} L} \boldsymbol{\theta}=\boldsymbol{\kappa} \hat{\boldsymbol{\theta}}_{\text {opt }}$, with

$\boldsymbol{\kappa}=\frac{\sigma_{d}{ }^{2}+\hat{\boldsymbol{\theta}}^{T} \mathbf{R}_{u}^{\hat{L} \hat{L}} \hat{\boldsymbol{\theta}}}{\sigma_{d}{ }^{2}+\boldsymbol{\theta}^{T} \mathbf{R}_{u}^{L \hat{L}} \hat{\boldsymbol{\theta}}}$ and $\hat{\boldsymbol{\theta}}_{\text {opt }}=\mathbf{R}_{u}^{\hat{L} \hat{L}^{-1}} \mathbf{R}_{u}^{\hat{L} L} \boldsymbol{\theta}$.

Replacing $\hat{\boldsymbol{\theta}}$ by $\boldsymbol{k} \hat{\boldsymbol{\theta}}_{\text {opt }}$ in the expression of $\boldsymbol{\kappa}$ yields:

$(1-\kappa) \sigma_{d}^{2}=0$.

One can then distinguish two cases, depending on whether $\sigma_{d}=0$. If $\sigma_{d}^{2} \neq 0$, then $\kappa=1$. To determine whether $\hat{\boldsymbol{\theta}}_{\text {opt }}$ is a maximum, we show that $r(\hat{\boldsymbol{\theta}})$ is concave in the neighbourhood of $\hat{\boldsymbol{\theta}}_{\text {opt }}$. Since $r(\hat{\boldsymbol{\theta}})$ is at least $C^{2}$ on $\mathfrak{R}^{\hat{L}}$, we can determine its Hessian matrix $\mathbf{H}$ :

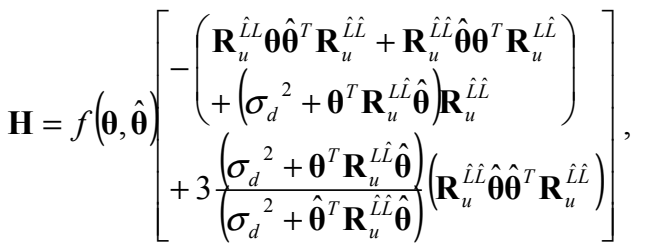

where $f(\boldsymbol{\theta}, \hat{\boldsymbol{\theta}})$ is a scalar-valued positive function. Thus $r(\hat{\boldsymbol{\theta}})$ is concave when:

$\hat{\boldsymbol{\theta}}^{T} \mathbf{H} \hat{\boldsymbol{\theta}} \leq 0 \Leftrightarrow g(\boldsymbol{\theta}, \hat{\boldsymbol{\theta}})=\sigma_{d}{ }^{2}\left(2 \hat{\boldsymbol{\theta}}^{T} \mathbf{R}_{u}^{\hat{L} \hat{L}} \hat{\boldsymbol{\theta}}-3 \boldsymbol{\theta}^{T} \mathbf{R}_{u}^{L \hat{L}} \hat{\boldsymbol{\theta}}-\sigma_{d}{ }^{2}\right) \leq 0$

We find:

$$
g\left(\boldsymbol{\theta}, \hat{\boldsymbol{\theta}}_{\text {opt }}\right)=-\boldsymbol{\sigma}_{d}{ }^{2}\left(\boldsymbol{\theta}^{T} \mathbf{R}_{u}^{L \hat{L}} \mathbf{R}_{u}^{\hat{L} \hat{L}^{-1}} \mathbf{R}_{u}^{\hat{L} L} \boldsymbol{\theta}+\boldsymbol{\sigma}_{d}{ }^{2}\right)<0 .
$$

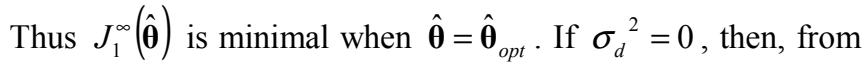
(13), $r(\hat{\boldsymbol{\theta}})$ is maximal and positive when $\hat{\boldsymbol{\theta}}=\boldsymbol{\kappa} \hat{\boldsymbol{\theta}}_{\text {opt }}, \forall \boldsymbol{\kappa}>0$. To pursue the analysis further, one can distinguish two more cases:

- $\quad \hat{L} \geq L$. It follows from (13) that $\hat{\boldsymbol{\theta}}_{\text {opt }}=\left.\boldsymbol{\theta}\right|_{\hat{L}}$. This proves Property 1.

- $\quad \hat{L}<L$. In general, $\hat{\boldsymbol{\theta}}_{\text {opt }} \neq\left.\boldsymbol{\theta}\right|_{\hat{L}}$. A notable exception is when $u$ is a white noise, in which case $\mathbf{R}_{u}^{\hat{L} \hat{L}}=\sigma_{u}^{2} \mathbf{I}$ and $\mathbf{R}_{u}^{\hat{L} L} \boldsymbol{\theta}=\left.\sigma_{u}^{2} \boldsymbol{\theta}\right|_{\hat{L}}$ imply $\hat{\boldsymbol{\theta}}_{\text {opt }}=\left.\boldsymbol{\theta}\right|_{\hat{L}}$, i.e. the first $\hat{L}$ coefficients of the impulse response are correctly identified. This proves Property 2.

Since $J_{1}^{\infty}(r)$ is decreasing and convex for $r \in[0,1]$ and $r$ is positive and concave in the neighbourhood of $\hat{\boldsymbol{\theta}}_{\text {opt }}\left(\operatorname{resp} . \boldsymbol{\kappa} \hat{\boldsymbol{\theta}}_{\text {opt }}\right.$ if $\left.\sigma_{d}^{2}=0\right), J_{1}^{\infty}(\hat{\boldsymbol{\theta}})$ is convex in a neighbourhood of $\hat{\boldsymbol{\theta}}_{\text {opt }}$ (resp. $\boldsymbol{k} \hat{\boldsymbol{\theta}}_{\text {opt }}$ ): this proves Property 3. Finally, because of the one-to-one correspondence between $J_{0}^{\infty}(r)$ and $J_{1}^{\infty}(r), J_{0}^{\infty}(\hat{\boldsymbol{\theta}})$ has the same minimum (or minima) as $J_{1}^{\infty}(\hat{\boldsymbol{\theta}})$ : this proves Property 4. $\square$

It is notable that $\hat{\boldsymbol{\theta}}_{\text {opt }}$ is independent of $\sigma_{b}$ : thus, Properties 1 to 4 hold regardless of the value of the variance of the measurement noise.

As a consequence of Property 3, it is sufficient to use a gradient algorithm to estimate the coefficients of the impulse response, provided a "good" initial guess is known (found with [5], for example) and the number of samples is "large enough". The corresponding gradient iteration is given by:

$\hat{\boldsymbol{\theta}}_{n+1}=\hat{\boldsymbol{\theta}}_{n}-\lambda_{n} \mathbf{g}\left(\hat{\boldsymbol{\theta}}_{n}\right), \lambda_{n}>0$,

$$
\left\{\begin{array}{l}
\mathbf{g}(\hat{\boldsymbol{\theta}})=\frac{1}{2} \frac{1}{\hat{\boldsymbol{\theta}}^{T} \mathbf{Q} \hat{\boldsymbol{\theta}}}\left[\left(\mathbf{P}-4 J_{1}^{N} \mathbf{Q}\right) \hat{\boldsymbol{\theta}}+\left(\mathbf{p}-4 J_{1}^{N} \mathbf{q}\right)\right] \\
\mathbf{P}(\hat{\boldsymbol{\theta}})=\sum_{l=1}^{N} \boldsymbol{\varphi}_{l, \hat{L}} \boldsymbol{\varphi}_{l, \hat{L}}^{T}\left(s_{l}-\hat{s}_{l}\right)^{2} \quad \mathbf{Q}=\sum_{l=1}^{N} \boldsymbol{\varphi}_{l, \hat{L}} \boldsymbol{\varphi}_{l, \hat{L}}^{T} . \\
\mathbf{p}(\hat{\boldsymbol{\theta}})=\sum_{l=1}^{N} \boldsymbol{\varphi}_{l, \hat{L}} d_{l}\left(s_{l}-\hat{s}_{l}\right)^{2} \quad \mathbf{q}=\sum_{l=1}^{N} \boldsymbol{\varphi}_{l, \hat{L}} d_{l}
\end{array}\right.
$$

A line-search method can be used to find an optimal value of the step parameter $\lambda_{n}$. Because of its simplicity, we have called the identification method based on the minimization of $J_{1}^{N}$ "BIMBO", for Basic Identification Method using Binary Observations. We show in Fig. 3 typical contour lines of $J_{0}^{256}$ and $J_{1}^{256}$ and the result of the gradient algorithm, when $\boldsymbol{\theta}^{T}=[1,1], \hat{L}=2, \sigma_{u}=1, \sigma_{b}=0.2$ and $\sigma_{d}=0$ or $\sigma_{d}=1$. All processes are white and Gaussian. Although the number of samples is large w.r.t. $L$, it is hardly conceivable to optimize $J_{0}^{256}$. 

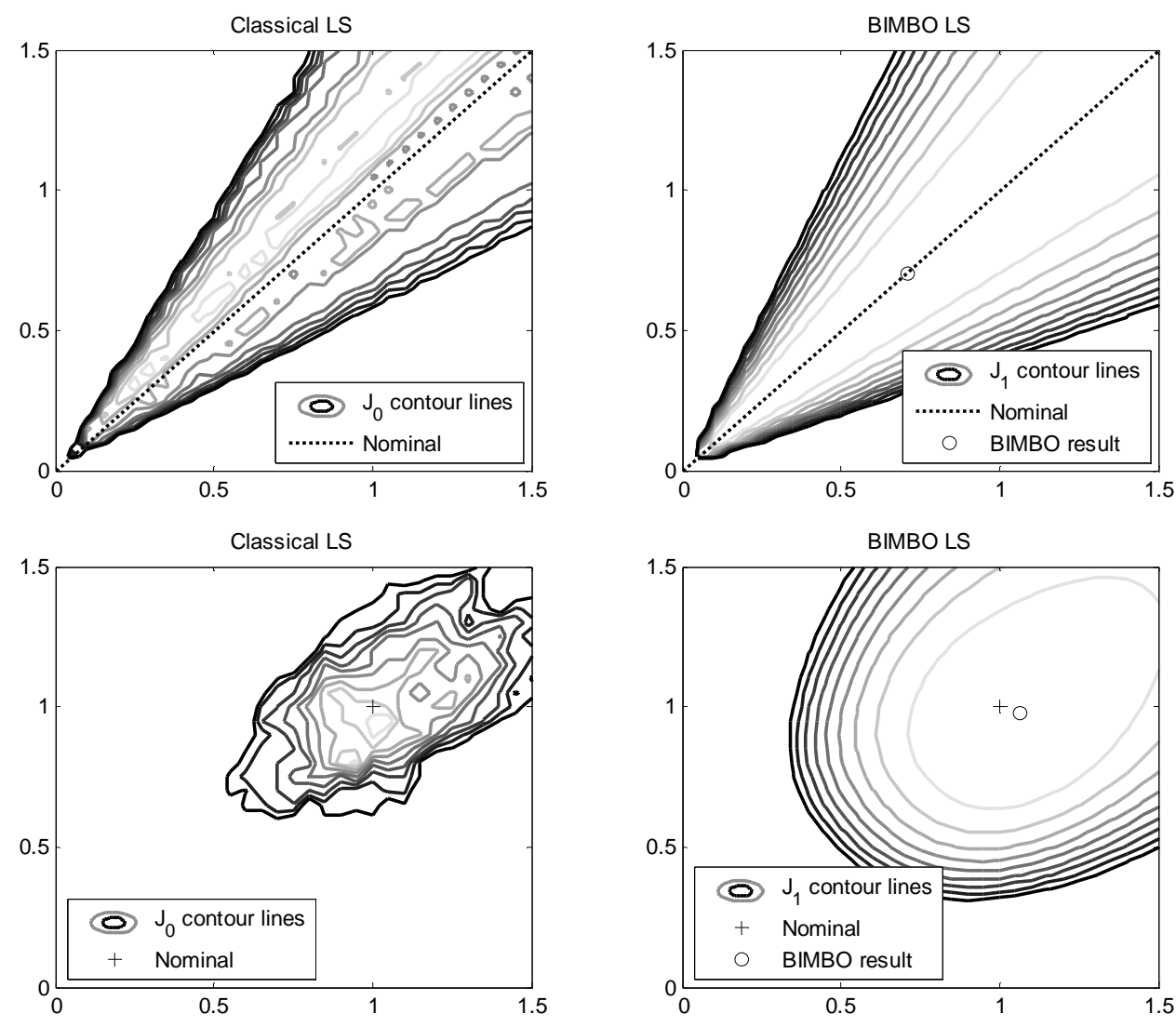

Fig. 3 - Contour lines of $J_{0}^{256}$ (left) and $J_{1}^{256}$ (right), when $\sigma_{d}=0$ (top) and $\sigma_{d}=1$ (bottom).

\section{CONCLUSION}

In this paper, we have introduced general WLS criteria for parameter estimation problems based on binary observations. Some asymptotical properties of this criterion were established in the simple case when $p=1$ (BIMBO method), under assumptions of Gaussianity. In particular, we have proved that this approach leads to consistent estimates of the system parameters, regardless of the variance of the measurement noise at the quantizer's input or of a dithering signal. Moreover, we have established that the corresponding criterion is locally convex, which makes it amenable to simple optimization tools. The extension of this approach to less simple systems (IIR or nonlinear) or to a framework of online identification is also being studied.

\section{REFERENCES}

[1] L Y Wang et al., "System identification using binary sensors", IEEE Transactions on Automatic Control, pp. 18921907, 2003

[2] L Ljung, "System identification - theory for the user", Prentice Hall, 1999

[3] E Walter, L Pronzato, "Identification of parametric models from experimental data", Springer, 1997

[4] M Negreiros et al., "Ultimate low cost analog BIST", 2003 Design Automation Conference, pp. 570-573, 2003

[5] J Juillard, E Colinet, "Initialization of the BIMBO self-test method using binary inputs and outputs", $46^{\text {th }}$ IEEE Conference on Decision and Control, pp. 161-166, 2008

[6] T Wigren, "Adaptive filtering using quantized output measurements", IEEE Transactions on Automatic Control, pp. 975-978, 1998

[7] L Y Wang et al., "Joint identification of plant rational models and noise distribution functions using binary-valued observations", Automatica, pp. 535-547, 2006

[8] Y Zhao et al., "Identification of Wiener systems with binary-valued output observations", Automatica, pp. 17521765, 2007

[9] E Rafaljowicz, "Linear systems identification from random threshold binary data", IEEE Transactions on Signal Processing, pp. 2064-2070, 1996

[10] E Rafaljowicz, "System identification from cheap, qualitative output observations", IEEE Transactions on Automatic Control, pp. 1381-1385, 1996

[11] E Bai, J Reyland, "Towards identification of Wiener systems with the least amount of a priori information on the nonlinearity", Automatica, pp. 910-919, 2008 